\title{
Boosting photonic quantum computation with moderate nonlinearity
}

\author{
A. Pick,,${ }^{1, \text { * }}$ E. S. Matekole,${ }^{2}$ Z. Aqua,${ }^{1}$ G. Guendelman, ${ }^{1}$ O. Firstenberg, ${ }^{3}$ J. P. Dowling,,${ }^{2, \dagger}$ and B. Dayan ${ }^{1}$ \\ ${ }^{1}$ Department of Chemical Physics, Weizmann Institute of Science, Rehovot 76100, Israel \\ ${ }^{2}$ Department of Physics and Astronomy, Louisiana State University, Baton Rouge, LA 70803, USA \\ ${ }^{3}$ Department of Physics, Weizmann Institute of Science, Rehovot 76100, Israel
}

\begin{abstract}
Photonic measurement-based quantum computation (MBQC) is a promising route towards faulttolerant universal quantum computing. A central challenge in this effort is the huge overhead in the resources required for the construction of large photonic clusters using probabilistic linear-optics gates. Although strong single-photon nonlinearity ideally enables deterministic construction of such clusters, it is challenging to realise in a scalable way. Here we explore the prospects of using moderate nonlinearity (with conditional phase shifts smaller than $\pi$ ) to boost photonic quantum computing and significantly reduce its resources overhead. The key element in our scheme is a nonlinear router that preferentially directs photonic wavepackets to different output ports depending on their intensity. As a relevant example, we analyze the nonlinearity provided by Rydberg blockade in atomic ensembles, in which the trade-off between the nonlinearity and the accompanying loss is well understood. We present protocols for efficient Bell measurement and GHZ-state preparation both key elements in the construction of cluster states, as well as for the CNOT gate and quantum factorization. Given the large number of entangling operations involved in fault-tolerant MBQC, the increase in success probability provided by our protocols already at moderate nonlinearities can result in a dramatic reduction in the required resources.
\end{abstract}

Photonic quantum computation is a leading platform in the effort towards fault-tolerant universal quantum computers [1]. It combines the paradigm of measurement-based quantum computation (MBQC) [511, where the computation is carried out by applying a sequence of measurements to entangled cluster states [12 14] with topological quantum error correction [15 20]. In particular, the promise of alloptical photonic quantum computation with discrete variables 21] lies in the ability to entangle single photons into graphs and clusters using only linear-optics probabilistic operations [22 29]. The price, however, is a huge overhead: constructing a cluster of $10^{7}$ photons (corresponding to $\sim 1000$ logical qubits, assuming $10^{4} \times$ redundancy for error correction) with probabilistic gates may require $10^{12}$ input single photons [30.

One approach to tackle this challenge is efficient and strong interaction with single quantum emitters, such as atoms, ions, or quantum dots. Such coupling ideally enables deterministic construction of cluster states either by generation of a stream of entangled photons 31 34, or by entangling single photons via photon-atom quantum gates [35 40]. However, achieving strong interaction with single quantum emitters requires challenging optical structures, which are not straightforwardly scalable. A number of theoretical works explored the possibility of enhancing weak Kerr-type nonlinearities by classical driving fields to make them strong enough to support photonic quantum computation [41 50]. To be precise, we define nonlinearity as "strong" if it can provide a conditional phase shift $\varphi=\pi$; namely, a difference of $\pi$ between twice the phase acquired by a single

\footnotetext{
* adi.pick@weizmann.ac.il

$\dagger$ Deceased 5 June, 2020.
}

photon in a mode, and the phase acquired by two photons in the same mode.

In contrast to previous studies, here we explore whether there is an intermediate regime between the linear optics and strong nonlinearity regimes in which moderate nonlinearity can provide a practical advantage. The motivation is that since fault-tolerant photonic quantum computation involves a large number of probabilistic gates [30, even a small improvement in the success probability per gate could amount to a dramatic reduction in the required resources. The reason for focusing on moderate nonlinearity is that attaining small phase shifts (e.g., $\pi / 10$ ) typically requires significantly less resources than attaining $\pi$, and, accordingly, is accompanied by much lower costs, such as, in particular, photon loss, which is the dominant fault mechanism in photonic qubits. Although advanced protocols for photonic quantum computing can tolerate up to $50 \%$ overall photon loss (being an inherently detectable error in most cases) [4, 17, 51, 52, the large number of elements and operations involved makes reaching this level challenging nonetheless. Given the progress in photonic technologies, including large scale on-chip single-photon detection capabilities with superconducting nanowires 53, 54, the additional loss induced by the nonlinear medium itself will likely become the dominant one. We therefore wish to quantify the trade-off offered by introducing nonlinear elements to the task of graph-states construction, namely increasing the success probability of each operation at the price of significant additional loss.

In order to take loss into account as accurately as possible, we consider below the very relevant and wellstudied platform of electromagnetically-induced transparency (EIT [55]) with Rydberg atoms (Rydberg- 


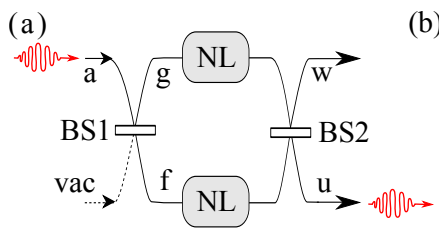

(b)
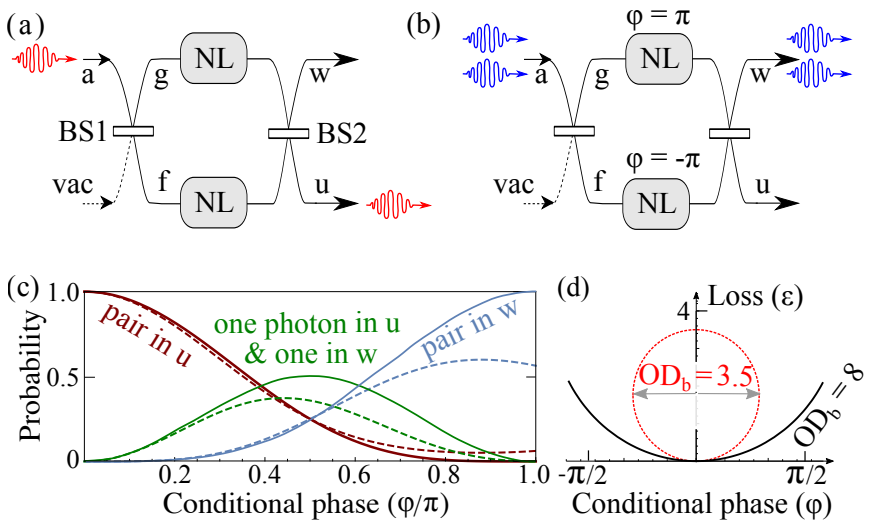

FIG. 1. Nonlinear router: (a) When a photon enters the interferometer in mode $a$, it leaves in $u$. (b) The interferometer contains a nonlinear medium (NL, gray) in both arms. Photon pairs traveling through different arms acquire opposite conditional phases $\pm \varphi$ [Eq. [2] ]. When $\varphi=\pi$, a pair that enters in mode $a$ leaves in mode $w$. (c) Probabilities for detection outcomes for an incoming pair in mode $a$ when $\varphi \in[0, \pi]$ : both photons in $u$ (brown), both in $w$ (blue), and one in each mode (green). Solid lines show a loss-free model [Eq. (3)]. Dashed lines include nonlinear loss in RydbergEIT systems [Eq. (6)]. We assume that the optical depth of the blockaded volume is $\mathrm{OD}_{\mathrm{b}}=30$, which sets the loss probability $\tau(\varphi)$ via Eq. (5), as shown in (d). The relation between the attenuation coefficient, $\varepsilon \equiv-\ln (1-\tau)$, and $\varphi$ is a circle [58], plotted for $\mathrm{OD}_{\mathrm{b}}=3.5$ and 8 (red and black).

EIT) [56, 57. In these systems, the loss grows quadratically with the conditional phase shift $\varphi$ (for small $\varphi$ ), which is eventually limited by the physical parameters of the Rydberg ensemble [58].

The key element in our protocols is a nonlinear router 59 61, realized by a Mach-Zehnder interferometer (MZI) with a nonlinear medium in both arms (Fig. 1). The conditional phase-shift acquired by two-photon pulses (photon pairs) in the nonlinear media causes the router to preferentially direct single photon pulses to one port and photon pairs to another. We use the router to obtain nonlinearity-enhanced protocols for Bell-state measurement (BM) and for Greenberger-Horne-Zeilinger (GHZ) state preparation (Figs. 2 and 3), which are key building blocks in MBQC [5. As an application, we use these elements to construct protocols for a CNOT gate and quantum factorization (Fig. 4). By adding nonlinearity, our protocols outperform the linear methods, potentially reducing resource requirements for faulttolerant photonic quantum computing by up to two orders of magnitude already at moderate nonlinearities.

Nonlinear router: As shown in Fig. 1(a), when single photons enter the MZI in mode $a$, they exit through port $u$, following the transformation rule [62]

$$
a^{\dagger} \stackrel{\mathrm{BS} 1}{\longrightarrow} 1 / \sqrt{2}\left(f^{\dagger}+i g^{\dagger}\right) \stackrel{\mathrm{BS} 2}{\longrightarrow} u^{\dagger} .
$$

The MZI contains a nonlinear atomic medium that induces opposite conditional phase shifts $\pm \varphi$ for photon pairs in each of its arms:

$$
\left(f^{\dagger}\right)^{2} \rightarrow e^{i \varphi}\left(f^{\dagger}\right)^{2} \quad, \quad\left(g^{\dagger}\right)^{2} \rightarrow e^{-i \varphi}\left(g^{\dagger}\right)^{2} .
$$

As a result, photon pairs undergo the transformation

$$
\left(a^{\dagger}\right)^{2} \stackrel{\mathrm{BS} 1}{\longrightarrow} \frac{1}{2}\left(e^{i \frac{\varphi}{2}} f^{\dagger}+i e^{-i \frac{\varphi}{2}} g^{\dagger}\right)^{2} \stackrel{\mathrm{BS} 2}{\longrightarrow}\left(w^{\dagger} \sin \frac{\varphi}{2}+u^{\dagger} \cos \frac{\varphi}{2}\right)^{2} .
$$

Consequently, the probability of routing pairs to the second output mode $w$ increases monotonously with $\varphi$ [Fig. 1(c)]. For $\varphi=\pi$, the nonlinear router deterministically separates pairs to a different port than single photons [Fig. 1(b)].

The effect of photon loss: Our schemes can be implemented with any nonlinear medium that provides moderate conditional phase shifts, including tightly confined atomic ensembles (e.g., atom-cladded optical fibers 63 65, atom-filled hollow-core fibers 66 68, or optical traps 69 71]) and nonlinear fibers [72. Here, we focus on Rydberg-EIT systems to analyze effect of loss on our protocols. We choose this platform since it enables achieving moderate (and even large) conditional phase shifts [56, 57, since linear losses under EIT conditions can be made negligible [55], and since the phase-loss relation in this system is well understood [58. In such systems, once a photon generates a Rydberg excitation, the energy levels of the surrounding atoms (within the Rydberg-blockade radius) are shifted, violating the EIT conditions [73]. Consequently, any subsequent photon in this volume acquires a phase and suffers loss.

We model loss as the annihilation of a photon (in $f$ or $g)$ and the creation of a photon in an undetected mode ( $\ell$ or $k$ ). Denoting the absorption probability by $\tau$, a photon pair in the atomic medium follows the rule:

$$
\begin{aligned}
f^{\dagger^{2}} & \rightarrow \sqrt{1-\tau} e^{i \varphi} f^{\dagger^{2}}+\sqrt{\tau} f^{\dagger} \ell^{\dagger} \\
g^{\dagger^{2}} & \rightarrow \sqrt{1-\tau} e^{-i \varphi} g^{\dagger^{2}}+\sqrt{\tau} g^{\dagger} k^{\dagger} .
\end{aligned}
$$

The conditional phase shift $\varphi$ and absorption coefficient $\varepsilon \equiv-\ln (1-\tau)$ follow the phase-loss circle [58]:

$$
\varphi^{2}+\left(\frac{\varepsilon}{2}-\frac{\mathrm{OD}_{\mathrm{b}}}{4}\right)^{2}=\left(\frac{\mathrm{OD}_{\mathrm{b}}}{4}\right)^{2},
$$

where $\mathrm{OD}_{\mathrm{b}}$ is the optical depth of the blockade volume - the core resource of nonlinearity in Rydberg-EIT systems [74. As evident from Eq. (5) and Fig. 11(d), high $\mathrm{OD}_{\mathrm{b}}$ enables large conditional phase shifts with low loss. In particular, $\mathrm{OD}_{\mathrm{b}}>4 \pi$ is required for $\varphi=\pi$. The value $\mathrm{OD}_{\mathrm{b}}=13$ has already been reached experimentally [75], and higher values have been predicted [76, 77]. Additionally, high total OD can be utilized to generate an effective cavity in the atomic medium with finesses $F \sim(\mathrm{OD} / 2)^{0.4}$ 58, leading to a phase-loss circle whose 

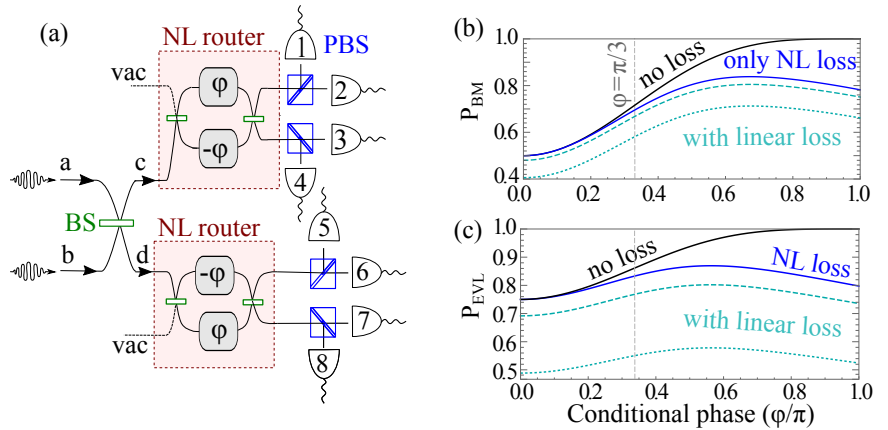

FIG. 2. Nonlinear Bell measurement (BM): Two photons are sent through a beam splitter (BS) and nonlinear routers before reaching polarizing beam splitters (PBS) followed by detectors (1-8), which measure their joint polarization state in the Bell basis. While $\left|\psi_{ \pm}\right\rangle$produce distinct final states, $\left|\phi_{ \pm}\right\rangle$can only be distinguished with nonlinearity. (b) Success probability of a BM, $P_{\mathrm{BM}}$, as a function of $\varphi$. Black solid curve neglects loss [Eq. (8) with $\tau=0$ and $P_{\mathrm{DE}}=1$ ]. Blue solid curve includes only nonlinear loss, assuming $\mathrm{OD}_{\mathrm{b}}=30$, while the cyan dashed (dotted) curve includes also linear loss with $P_{\mathrm{DE}}=98 \%$ (90\%). With $P_{\mathrm{DE}}=98 \%$ and $\varphi=\pi / 3, P_{\mathrm{BM}}$ improves from 0.48 to 0.67 . (c) Success probability of the nonlinear enhancement of the Ewert-van Loock protocol [78, $P_{\text {EVL }}$ [Eq. [9] ] that includes two ancillary qubits. Curves are labeled as in (b). With $P_{\mathrm{DE}}=98 \%$ and $\varphi=\pi / 3, P_{\mathrm{EVL}}$ improves from 0.69 to 0.77 .

radius is $F$ times larger, thereby enabling an effective $\mathrm{OD}_{\mathrm{b}}$ of 100 or more. Using Eqs. $(1,4,5)$, we obtain

$$
\begin{array}{r}
a^{\dagger^{2}} \rightarrow \frac{\sqrt{1-\tau(\varphi)}}{2}\left[\cos \varphi\left(w^{\dagger^{2}}-u^{\dagger^{2}}\right)-2 \sin \varphi\left(w^{\dagger} u^{\dagger}\right)\right] \\
-\frac{1}{2}\left(w^{\dagger^{2}}+u^{\dagger^{2}}\right)+\frac{\sqrt{\tau(\varphi)}}{2 \sqrt{2}}\left[\left(w^{\dagger}+i u^{\dagger}\right) \ell^{\dagger}-\left(u^{\dagger}+i w^{\dagger}\right) k^{\dagger}\right] .
\end{array}
$$

From Eq. (6), we calculate the probabilities for different outcomes of the MZI when including loss [Fig. 11(c)].

Equations (56) assume that the photon wavelength is tuned exactly on the EIT resonance, where the singlephoton phase shift and loss are ideally zero. In the supplementary material (SM), we analyze the possibility of detuning from the EIT resonance to include the effect of single-photon phase shift and loss in the atomic medium. We find that the performance of our protocols is relatively unaffected by these processes.

Nonlinear Bell-state measurement (BM): As a first application, we use the nonlinear router to improve linear BMs. We begin this section by revisiting the traditional linear BM protocol. Then, we present our nonlinear protocol and discuss its key features in the limit of strong nonlinearity and negligible loss. Finally, we discuss moderate nonlinearity and non-negligible loss.

A photonic qubit is defined as a single excitation in an arbitrary coherent combination of two non-overlapping optical modes. For convenience, here we use the linear polarization basis, yet our analysis is generally applicable to other choices of optical modes, including dual-rail and time-bin qubits. In this basis, the photonic twoqubit Bell states are $\left|\psi_{ \pm}\right\rangle=\frac{1}{\sqrt{2}}\left(a_{H}^{\dagger} b_{V}^{\dagger} \pm a_{V}^{\dagger} b_{H}^{\dagger}\right)|\mathrm{vac}\rangle$ and $\left|\phi_{ \pm}\right\rangle=\frac{1}{\sqrt{2}}\left(a_{H}^{\dagger} b_{H}^{\dagger} \pm a_{V}^{\dagger} b_{V}^{\dagger}\right) \mid$ vac $\rangle$, where $x_{k}^{\dagger}$ denotes the creation operator of a photon in mode $x$ with polarization $k$ operating on the vacuum state $|\mathrm{vac}\rangle$. In a linear-optics BM, photons are sent through a balanced beam splitter (BS) 79]. Applying the BS transformation $a_{k}^{\dagger} \rightarrow \frac{1}{\sqrt{2}}\left(d_{k}^{\dagger}+i c_{k}^{\dagger}\right)$ and $b_{k}^{\dagger} \rightarrow \frac{1}{\sqrt{2}}\left(c_{k}^{\dagger}+i d_{k}^{\dagger}\right)$, one finds

$$
\begin{gathered}
\left|\psi_{-}\right\rangle \stackrel{\mathrm{BS}}{\longrightarrow} \frac{1}{\sqrt{2}}\left(d_{H}^{\dagger} c_{V}^{\dagger}-c_{H}^{\dagger} d_{V}^{\dagger}\right)|\mathrm{vac}\rangle \\
\left|\psi_{+}\right\rangle \stackrel{\mathrm{BS}}{\longrightarrow} \frac{i}{\sqrt{2}}\left(d_{H}^{\dagger} d_{V}^{\dagger}+c_{H}^{\dagger} c_{V}^{\dagger}\right)|\mathrm{vac}\rangle \\
\left|\phi_{ \pm}\right\rangle \stackrel{\mathrm{BS}}{\longrightarrow} \frac{i}{2 \sqrt{2}}\left[\left(d_{H}^{\dagger}\right)^{2}+\left(c_{H}^{\dagger}\right)^{2} \pm\left(\left(d_{V}^{\dagger}\right)^{2}+\left(c_{V}^{\dagger}\right)^{2}\right)\right]|\mathrm{vac}\rangle .
\end{gathered}
$$

The states $\left|\psi_{ \pm}\right\rangle$lead to distinguishable outcomes. While $\left|\psi_{-}\right\rangle$produces one photon in $c_{k}$ and one in $d_{k}$, the state $\left|\psi_{+}\right\rangle$produces an orthogonal pair in either $c_{k}$ or $d_{k}$. In contrast, the states $\left|\phi_{ \pm}\right\rangle$produce a "bunched" pair in one of the four detectors and are, therefore, indistinguishable. Hence, when detectors are placed at the exit of the BS, the success probability of the BM is $50 \%[79$.

By adding nonlinearity, one can improve the success probability of the BM. To this end, we place nonlinear routers that help distinguish between $\left|\phi_{ \pm}\right\rangle$at the output of the BS [red boxes in Fig. 22(a)]. When existing the routers, the photon pass through polarizing beam splitters (PBSs), which transmit horizontal and reflect vertical polarization, before hitting single-photon detectors (labeled as 1-8). When the acquired conditional phase shift in the MZIs is $\varphi=\pi$ and when neglecting loss, all four Bell states are distinguishable by this setup. That is, each input Bell state produces a unique set of detection clicks. For example, only the state $\left|\psi_{+}\right\rangle$ can produce clicks in $3 \& 4$ or $5 \& 6$ (see SM) 80].

Next, we consider the effect of photon loss on our scheme, caused either by the nonlinear medium (NL loss) or by the detectors. In the SM, we compute the success probability in the presence of loss and obtain

$$
P_{\mathrm{BM}}=P_{\mathrm{DE}}^{2}\left[1-\frac{1}{8}(\sqrt{1-\tau(\varphi)} \cos \varphi+1)^{2}-\frac{\tau(\varphi)}{4}\right],
$$

where $P_{\mathrm{DE}}$ denotes the single-photon detection efficiency. The formula is evaluated in Fig. 2(b). Our scheme assumes multiple detectors or photon-number resolving detectors are used 81,84 to distinguish between instances with two photons in the same detector from instances with a single detection due to a loss event. Assuming moderate conditional phase shift of $\varphi=\pi / 3$ (achievable using available setups [85]) and $P_{\mathrm{DE}}=98 \%$ improves the $P_{\mathrm{BM}}$ from 0.48 to 0.67 . Evidently, in the presence of loss, the optimal operating point is at an intermediate phase $\varphi_{\mathrm{opt}}<\pi$, which tends to $\pi$ upon increasing $\mathrm{OD}_{\mathrm{b}}$, scaling as $\pi-\varphi_{\text {opt }} \propto \mathrm{OD}_{\mathrm{b}}^{-1}$ at large $\mathrm{OD}_{\mathrm{b}}$ values (Fig. $\mathrm{D} 1$ in the $\mathrm{SM}$ ). 


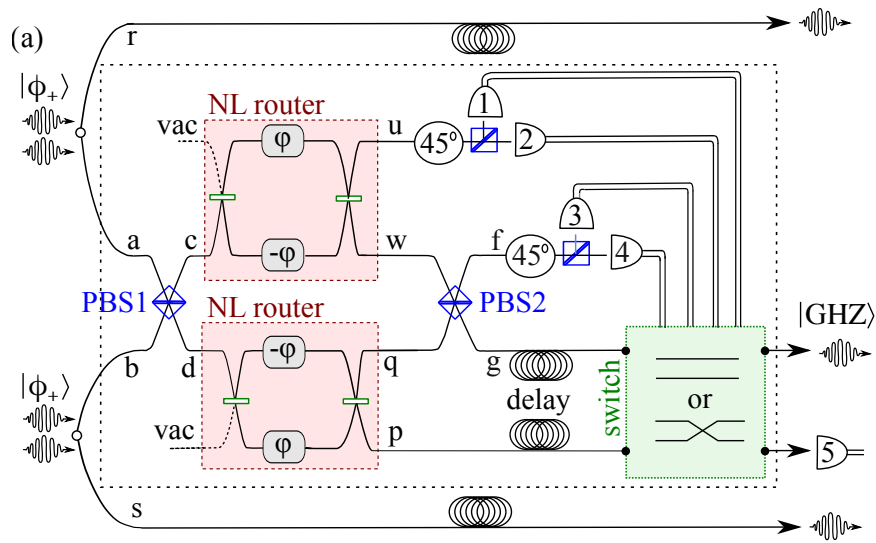

(b)

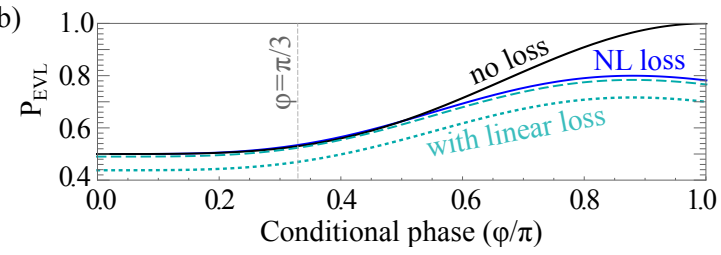

FIG. 3. Nonlinear GHZ state generation: (a) Two Bell pairs are prepared, and one photon from each pair is sent through PBS1. Two scenarios can produce a GHZ state: 1. When photons leave PBS1 through different ports and exit the routers in ports $u$ and $p$. 2. When photons leave PBS1 through the same port and also exit the routers in $w$ or $q$. When none of the photons are lost, zero or two clicks in detectors $1-4$ or a click in 5 herald failure. The switch selects one of the output modes, $g$ or $p$, that together with modes $r$ and $s$ contain a three-photon GHZ state when our protocol succeeds; during its operation, photons travel in delay lines (coils). (b) Success probability, $P_{\mathrm{GHZ}}$, as function of $\varphi$. Black solid curve neglects loss [Eq. 110 with $\tau=0$ and $P_{\mathrm{DE}}=1$ ]. Blue solid curve includes only nonlinear loss, assuming $\mathrm{OD}_{\mathrm{b}}=30$, while cyan dashed (dotted) curve includes also detector loss with $P_{\mathrm{DE}}=98 \%$ (90\%). With $P_{\mathrm{DE}}=98 \%$ and $\varphi=\pi / 3, P_{\mathrm{GHZ}}$ improves from 0.49 to 0.52 .

Figure 2(c) shows our nonlinear modification of the linear Ewert-van Loock protocol, that attains higher success probabilities at the cost of using two ancillary qubits [78, 86]. As shown in the SM, the success probability of the protocol is

$$
P_{\mathrm{EVL}}=P_{\mathrm{DE}}^{4}\left[1-\frac{1}{16}(\sqrt{1-\tau(\varphi)} \cos \varphi+1)^{2}-\frac{\tau(\varphi)}{4}\right]
$$

Assuming $\pi / 3$ and $P_{\mathrm{DE}}=98 \%$, the success probability $P_{\mathrm{EVL}}$ improves from 0.69 to 0.77 .

Nonlinear GHZ-state preparation: Our protocol is shown in Fig. 3(a). We discuss its key features here and provide the derivation details in the SM. Initially, two Bell states are prepared in $\left|\phi_{+}\right\rangle\left|\phi_{+}\right\rangle$and one photon from each Bell pair is sent through a polarizing beam splitter (PBS1). Then, the photons enter nonlinear routers. The photons may either leave PBS1 from different ports $\left(c_{H}^{\dagger} d_{H}^{\dagger}\right.$ and $\left.c_{V}^{\dagger} d_{V}^{\dagger}\right)$ or through the same port $\left(c_{H}^{\dagger} c_{V}^{\dagger}\right.$ and $\left.d_{H}^{\dagger} d_{V}^{\dagger}\right)$. In the former case, the photons leave the routers in modes $u$ and $p$. Then, the photon in $u$ undergoes a $45^{\circ}$ rotation and a subsequent measurement of the rotated photon in the diagonal basis projects the surviving photons onto a GHZ state. This process is called "fusion type I" 23.

Our nonlinear scheme aims to "save" also photons that leave PBS1 through the same port. To this end, we use nonlinear routers that have the property that when $\varphi=\pi$, photons from $c_{H}^{\dagger} c_{V}^{\dagger}$ and $d_{H}^{\dagger} d_{V}^{\dagger}$ are routed into modes $w_{H}^{\dagger} w_{V}^{\dagger}$ and $q_{H}^{\dagger} q_{V}^{\dagger}$. By sending these photons to PBS2, rotating the photon in mode $f$, and measuring the rotated photon in the diagonal basis, a GHZ state is produced. By adding the probabilities for successful GHZ-state generation, either by "fusion type I" or by successful nonlinear routing, we obtain (see SM)

$$
P_{\mathrm{GHZ}}=P_{\mathrm{DE}}\left[\frac{1}{2}+\frac{1}{8}(\sqrt{1-\tau(\varphi)} \cos \varphi-1)^{2}\right] .
$$

$P_{\mathrm{GHZ}}$ as a function of $\varphi$ is shown in Fig. 3(b). The optimal operating point is attained at $\varphi_{\mathrm{opt}}<\pi$ and scales as $\pi-\varphi_{\mathrm{opt}} \propto \mathrm{OD}_{\mathrm{b}}^{-1 / 3}$ with increasing $\mathrm{OD}_{\mathrm{b}}$ (Fig. D1). With $P_{\mathrm{DE}}=98 \%$ and $\varphi=\frac{\pi}{3}, P_{\mathrm{GHZ}}$ improves from 0.49 to 0.52 .

Our protocol accepts four input photons and (when successfully operated) produces three output photons in a GHZ state. The goal of the switch is to select the modes that contain the GHZ state in order to prepare the input for a quantum computation protocol or for a subsequent stage in a cluster-state-generation protocol. Specifically, the switch selects to output mode $g$ and measure $p$ if there was a click in detectors 3 or 4 , and vice versa if a click occurred in detectors 1 or 2 . During the switch operation, the photons travel in delay lines. Success of this scheme is heralded only if exactly one photon is detected - by detectors 1-4. Failure is heralded by detection of two photons in detectors 1-5 (indicating that the output is empty), or by the lack of any detection events in detectors 1-4 (whether cause by imperfect routing, detection inefficiency or loss). All these cases are accordingly disregarded. If two photons are routed to detectors 1-5 but only one is detected due to loss (e.g. in the delay lines or the switch) or imperfect detection efficiency, this leads to a "false positive" indication of success. However, the result in this case is again the lack of photon at the output channel, and therefore is equivalent to any other loss event, and is accordingly tolerated as long as the overall loss is below the required threshold.

CNOT and Factorization: In Ref. [5, Gottesman and Chuang (GC) present an optical-circuit implementation of the CNOT gate, which requires two GHZ states and three BMs [Fig. 4(a)]. Accordingly, the success probability of this protocol scales like $\left(P_{\mathrm{GHZ}}\right)^{2} \times\left(P_{\mathrm{BM}}\right)^{3}$, being $1 / 2^{5}$ in the linear-optics case. When using our 
(a)
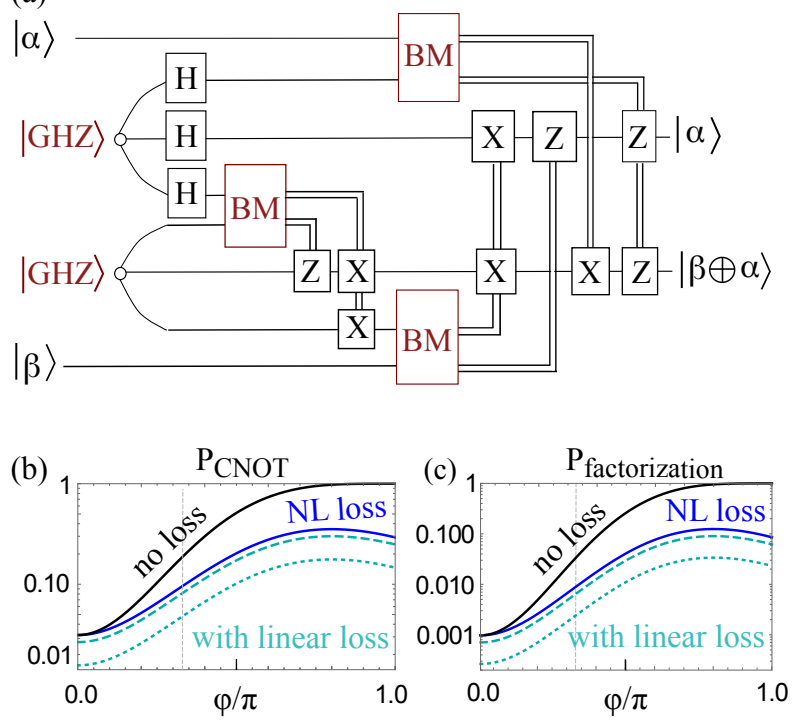

FIG. 4. Nonlinear CNOT and quantum factorization: (a) The Gottesman-Chuang linear CNOT gate [5]. The gate requires three BMs, two GHZ states and single-qubit Pauli $(X, Z)$ and Hadamard $(H)$ rotations. (b) Success probability (log scale) of the CNOT gate, using our nonlinear protocol (with BMs and GHZs from Figs. 2-3). Black solid curve neglects loss [Eq. 10 with $\tau=0$ and $P_{\mathrm{DE}}=1$ ]. Blue solid curve includes only nonlinear loss, assuming $\mathrm{OD}_{\mathrm{b}}=$ 30, while cyan dashed (dotted) curve includes also detector loss with $P_{\mathrm{DE}}=98 \%$ (90\%). (c) Success probability (log scale) of quantum factorization of the number 15, using a protocol that requires two CNOT gates [87, with the same building blocks and curve labels as in (b). For $\varphi=\pi / 3$ and $P_{\mathrm{DE}}=98 \%$, the success probabilities of CNOT and quantum factorization increase by factors of 3.32 and 11 respectively.

nonlinear elements, the success probability for $\varphi=\pi / 3$ becomes $0.72^{3} * 0.53^{2}=0.105$, which is 3.32 larger than $1 / 2^{5}$, as shown in Fig. 4(c) 88. As CNOT is an elementary building block in most quantum protocols, this enhancement is a dramatic result. For example, the algorithm for quantum factorization of the number 15 from Ref. [87] requires two CNOT gates. Accordingly, our nonlinear protocols lead to an order-ofmagnitude (11-fold) improvement in its success probability at $\varphi=\pi / 3$ [Fig. $4(\mathrm{~d})]$.

Discussion: We examined photonic quantum computation protocols in the intermediate regime between linear optics and strong nonlinearity at the single photon level, and presented efficient protocols for key elementary operations, including BM, GHZ-state generation, CNOT gate, and quantum factorization. Our results demonstrate the potential of moderate nonlinearity, which is achievable in a variety of platforms, using Rydberg-EIT systems [57, 89] as a relevant example. As photonic quantum computation, and faulttolerant MBQC in particular, require a large number of elementary operations [30], any modest increase in the success probability of each operation is translated to a dramatic reduction in the required resources. For example, a conditional phase shift of $\varphi=\pi / 3$, which in our scheme increases the success probability of ancillaassisted BM with detection efficiency of $98 \%$ from 0.69 to 0.77 , and of GHZ-state preparation from 0.49 to 0.52 , can be translated into two orders of magnitude reduction in resources after 35 operations. With the recent developments in interacting atomic ensembles with integrated photonics 63 65, 69 71, 90, 91, few-photon nonlinearty on chip-scale devices is becoming feasible, making protocols that rely on moderate nonlinearities a promising new platform for photonic quantum information processing.

Acknowledgment. The authors thank Ephraim Shahmoon, Serge Rosenblum, and Ran Finkelstein for helpful discussions. AP acknowledges support of the Koshland Foundation. BD and OF acknowledge support from the Israeli Science Foundation. ESK, BD, and JPD are also supported by Binational Science Foundation. ESM and JPD acknowledge the support by the U.S. Air Force Office Scientific Research, and also by the U.S. Army Research Office with the grant W911NF17-1-0541. BD is also supported by the Minerva Foundation, IMOD (OR RISHON), and a research grant from Dr. Saul Unter. OF is also supported by the European Research Council starting investigator grant QPHOTONICS 678674. 


\section{SUPPLEMENTARY MATERIAL}

\section{TABLE OF CONTENTS}

A. Nonlinear Bell measurement

A1. Protocol without ancillas.

A2. Protocol with ancillas.

B. Nonlinear GHZ-state preparation.

C. Introducing single-photon phase shifts

C1. Nonlinear router

C2. Nonlinear Bell measurement.

C3. Nonlinear GHZ-state generation.

D. Selecting optimal phase shifts.

\section{A: Nonlinear Bell measurement}

\section{Protocol without ancillas}

In this section, we provide calculation details for the success probability of a nonlinear Bell measurement [Eq. (8) in the main text]. For convenience, we present Fig. 2(a) from the main text in Fig. A.1, and introduce additional labeling of the modes involved in the protocol. First, we rewrite Eq. (7) in the diagonal basis

$$
\begin{gathered}
\left|\psi_{+}\right\rangle \stackrel{\mathrm{BS}}{\longrightarrow} \frac{i}{2 \sqrt{2}}\left[\left(c_{+}^{\dagger}\right)^{2}-\left(c_{-}^{\dagger}\right)^{2}+\left(d_{+}^{\dagger}\right)^{2}-\left(d_{-}^{\dagger}\right)^{2}\right]|\mathrm{vac}\rangle \\
\left|\phi_{+}\right\rangle \stackrel{\mathrm{BS}}{\longrightarrow} \frac{i}{2 \sqrt{2}}\left[\left(c_{+}^{\dagger}\right)^{2}+\left(c_{-}^{\dagger}\right)^{2}+\left(d_{+}^{\dagger}\right)^{2}+\left(d_{-}^{\dagger}\right)^{2}\right]|\mathrm{vac}\rangle \\
\left|\phi_{-}\right\rangle \stackrel{\mathrm{BS}}{\longrightarrow} \frac{i}{\sqrt{2}}\left[c_{+}^{\dagger} c_{-}^{\dagger}+d_{+}^{\dagger} d_{-}^{\dagger}\right]|\mathrm{vac}\rangle .
\end{gathered}
$$

To trace the evolution upon entering and leaving the MZI, we invoke the beam-splitter (BS) transformation

$$
\begin{aligned}
c^{\dagger} & \rightarrow \frac{1}{\sqrt{2}}\left(f^{\dagger}+i g^{\dagger}\right) \\
f^{\dagger} & \rightarrow \frac{1}{\sqrt{2}}\left(w^{\dagger}+i u^{\dagger}\right) \\
g^{\dagger} & \rightarrow \frac{1}{\sqrt{2}}\left(u^{\dagger}+i w^{\dagger}\right) .
\end{aligned}
$$

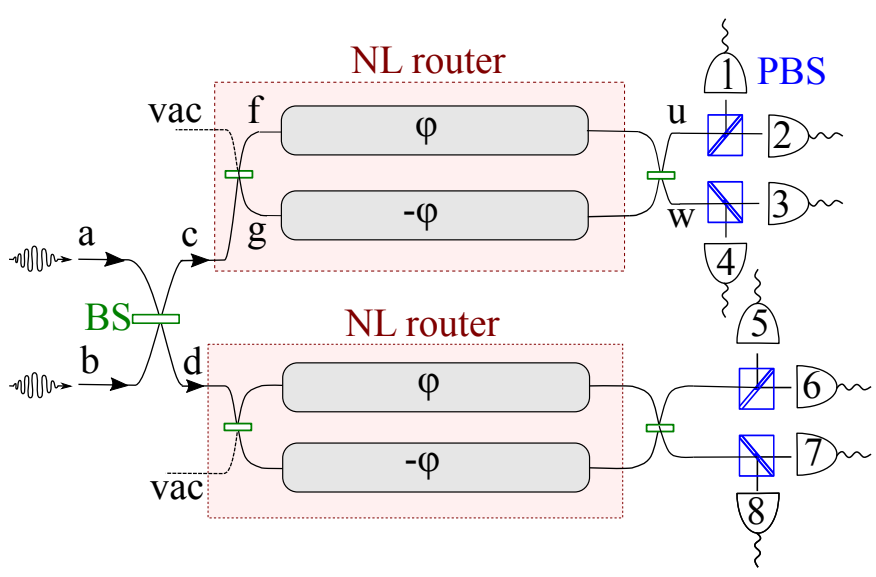

FIG. A.1. For convenience, we present Fig. 2(a) from the main text, introducing labeling of the modes inside the interferometer (modes $f, g, u$ and $w$ ).
Due to self-phase modulation in the atomic medium, an identical photon pair that enters modes $f$ or $g$ undergoes the transformation

$$
\begin{aligned}
& \left(f_{ \pm}^{\dagger}\right)^{2} \rightarrow \sqrt{1-\tau} e^{i \varphi}\left(f_{ \pm}^{\dagger}\right)^{2}+\sqrt{\tau} f_{ \pm}^{\dagger} \ell_{ \pm}^{\dagger} \\
& \left(g_{ \pm}^{\dagger}\right)^{2} \rightarrow \sqrt{1-\tau} e^{i \varphi}\left(g_{ \pm}^{\dagger}\right)^{2}+\sqrt{\tau} g_{ \pm}^{\dagger} k_{ \pm}^{\dagger},
\end{aligned}
$$

where the first term on the right hand side accounts for phase acquisition and the second for loss. By using Eqs. A.1 A.4, we find

$$
\begin{gathered}
\left(c_{ \pm}^{\dagger}\right)^{2} \rightarrow \frac{\sqrt{1-\tau} \cos \varphi-1}{2}\left(w_{ \pm}^{\dagger}\right)^{2}-\frac{\sqrt{1-\tau} \cos \varphi+1}{2}\left(u_{ \pm}^{\dagger}\right)^{2}- \\
\sqrt{1-\tau} \sin \varphi w_{ \pm}^{\dagger} u_{ \pm}^{\dagger}+\frac{\sqrt{\tau}}{2 \sqrt{2}}\left[\left(w_{ \pm}^{\dagger}+i u_{ \pm}^{\dagger}\right) l_{ \pm}^{\dagger}-\left(u_{ \pm}^{\dagger}+i w_{ \pm}^{\dagger}\right) k_{ \pm}^{\dagger}\right],
\end{gathered}
$$

generalizing Eq. (6) in the main text. A similar transformation rule applies for the $d_{+} d_{-}$component of the wavefunction.

Both the states $\left|\phi_{+}\right\rangle$and $\left|\psi_{+}\right\rangle$are affected by the nonlinearity, since both contain identical photon pairs [see Eq. A.1]]. However, whenever two photons are detected, $\left|\phi_{+}\right\rangle$is distinguishable from the remaining states because it is the only state that produces a pair of orthogonal photons either in the upper detectors (1-4) or in the lower detectors (5-8). Therefore, when both photons reach the detectors, only the cases where both photons from $\left|\psi_{+}\right\rangle$are routed into $u_{+}^{\dagger} u_{-}^{\dagger}$ lead to failure. Therefore, the failure probability is found by collecting the terms proportional to $u_{+}^{\dagger} u_{-}^{\dagger}$. Photon loss also leads to failure of the BM. Both $\left|\phi_{+}\right\rangle$and $\left|\psi_{+}\right\rangle$can experience loss, and the final states in that case are indistinguishable. Given that the initial state is $\left|\psi_{+}\right\rangle$or $\left|\phi_{+}\right\rangle$, the probability for loss is $\tau / 2$. Therefore, the overall success probability becomes

$$
P_{\mathrm{BM}}=P_{\mathrm{DE}}^{2}\left[1-\frac{1}{8}(\sqrt{1-\tau(\varphi)} \cos \varphi+1)^{2}-\frac{\tau(\varphi)}{4}\right],
$$

where the last term corresponds to nonlinear-loss events and $P_{\mathrm{DE}}$ accounts for finite detection efficiency.

\section{Protocols with ancillas}

In the main text, we present a Bell measurement (BM) that uses conditional phase shifts to improve a linear method whose success probability is $50 \%$. However, linear protocols that use ancillary photons may have higher success rates, with probability approaching $100 \%$; the failure rate drops exponentially with the number of ancillary photons [86]. Fig. 2(c) depicts the success rate of a refined nonlinear protocol, which is based on a linear method with two ancillary qubits and success probability of $75 \%$ 78. The linear protocol and its nonlinear improvement are shown in Fig. A.2 (a) and (b) respectively. 
(a)

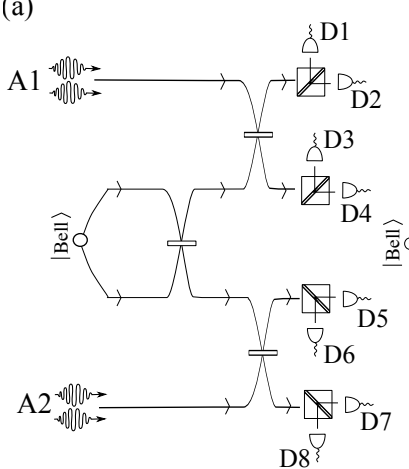

(b)

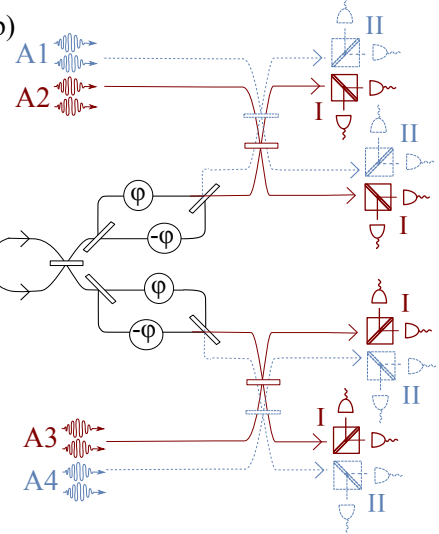

FIG. A.2. (a) Bell-state measurement using four ancillary qubits prepared in $\frac{1}{2}\left[\left(a_{H}^{\dagger}\right)^{2}+\left(a_{V}^{\dagger}\right)^{2}\right] \mid$ vac $\rangle$. A Bell state is sent through the device, and the final measurement can determine the input state with success probability of $75 \%$. (b) By introducing nonlinear routers (and additional ancillas and detectors), the success probability increases, and reaches $100 \%$ for $\pi$ conditional phase shifts, as shown by the green line in Fig. 2(b) in the main text.

The linear method, proposed independently by Grice and Ewert and van-Loock 78, 86, utilizes additional photons prepared in $\left|A_{i}\right\rangle=\frac{1}{2}\left[\left(a_{i, H}^{\dagger}\right)^{2}+\left(a_{i, V}^{\dagger}\right)^{2}\right] \mid$ vac $\rangle$, where the index $i=1,2$ enumerates the ancillas and $|v a c\rangle$ is the vacuum of the acillary modes. The protocol is shown inFig. A.2(a). we briefly revise the linear method before introducing our nonlinear modification. By using 8 detectors, the Bell state can be determined with success probability of $75 \%$. The states $\psi_{ \pm}$and $\phi_{ \pm}$ differ in the parity of $H$ and $V$ polarized photons, since the parity is unaltered by the device. The states $\psi_{ \pm}$ can be distinguished since they differ in the number of photons that reach the upper detectors $(D 1, \ldots, D 4)$. Finally, the states $\phi_{ \pm}$can only be distinguished with probability $50 \%$ (instances where all detected photons have the same polarization can occur for both $\phi_{+}$and $\phi_{-}$and lead to failure).

By introducing two nonlinear routers, one in each output of the first beam splitter, we increase the probability to distinguish between the $\phi_{ \pm}$states. The setup is shown in Fig. A.2(b). The success probability of our nonlinear protocol (when neglecting loss) is

$$
P_{\mathrm{EVL}}=P_{\mathrm{DE}}^{4}\left[1-\frac{1}{16}(\sqrt{1-\tau(\varphi)} \cos \varphi+1)^{2}-\frac{\tau(\varphi)}{4}\right]
$$

With a conditional phase shift of $\pi$, the BM becomes deterministic. Note, however, that the nonlinear setup requires four ancillary qubits and eight detectors (while the linear counterpart required only two ancilla qubits).

\section{B: Nonlinear GHZ-state preparation}

In the main text, we describe a nonlinear protocol for GHZ-state generation. In this section, we provide the details of the derivation. For the the notation we refer to Fig. B.1. The protocol uses a resource of two Bell pairs,

$$
|\psi\rangle=\frac{1}{2}\left(r_{H}^{\dagger} a_{H}^{\dagger}+r_{V}^{\dagger} a_{V}^{\dagger}\right)\left(s_{H}^{\dagger} b_{H}^{\dagger}+s_{V}^{\dagger} b_{V}^{\dagger}\right)|\mathrm{vac}\rangle .
$$

Then, one photon from each pair is sent through a polarizing beam splitter (PBS) (which transmits $H$-polarized photons and reflects $V$ photons) and the state becomes

$$
|\psi\rangle \stackrel{\text { PBS1 }}{\longrightarrow} \frac{1}{2}\left(r_{H}^{\dagger} d_{H}^{\dagger}+i r_{V}^{\dagger} c_{V}^{\dagger}\right)\left(s_{H}^{\dagger} c_{H}^{\dagger}+i s_{V}^{\dagger} d_{V}^{\dagger}\right)|\operatorname{vac}\rangle .
$$

This wavefunction contains two types of components: ones with single photons in each output arm of the PBS $\left(c_{H}^{\dagger} d_{H}^{\dagger}\right.$ and $\left.c_{V}^{\dagger} d_{V}^{\dagger}\right)$ and others with both photons in the same arm $\left(c_{H}^{\dagger} c_{V}^{\dagger}\right.$ and $\left.d_{H}^{\dagger} d_{V}^{\dagger}\right)$. The former type of terms lead to successful GHZ-state generation. For these states, our protocol reproduces the linear protocol "fission I." For these terms, the photons leave the routers through ports $u$ or $p$. Then, the photon in $u$ undergoes a $45^{\circ}$ rotation, resulting in

$$
\begin{aligned}
& \frac{1}{2}\left(r_{H}^{\dagger} s_{H}^{\dagger} c_{H}^{\dagger} d_{H}^{\dagger}-r_{V}^{\dagger} s_{V}^{\dagger} c_{V}^{\dagger} d_{V}^{\dagger}\right) \stackrel{\mathrm{MZI}}{\longrightarrow} \frac{1}{2}\left(r_{H}^{\dagger} s_{H}^{\dagger} p_{H}^{\dagger} u_{H}^{\dagger}-r_{V}^{\dagger} s_{V}^{\dagger} p_{V}^{\dagger} u_{V}^{\dagger}\right) \\
& \stackrel{45^{\circ}}{\longrightarrow} \frac{1}{2 \sqrt{2}}\left[u_{+}^{\dagger}\left(r_{H}^{\dagger} s_{H}^{\dagger} p_{H}^{\dagger}-r_{V}^{\dagger} s_{V}^{\dagger} p_{V}^{\dagger}\right)+u_{-}^{\dagger}\left(r_{H}^{\dagger} s_{H}^{\dagger} p_{H}^{\dagger}+r_{V}^{\dagger} s_{V}^{\dagger} p_{V}^{\dagger}\right)\right] .
\end{aligned}
$$

Therefore, a click in either $u_{+}$or $u_{-}$projects the surviving photons onto a GHZ state.

On the other hand, our protocol can save also the latter type of terms. The wavefunction components with both photons in the same arm (containing $c_{H}^{\dagger} c_{V}^{\dagger}$ and $\left.d_{H}^{\dagger} d_{V}^{\dagger}\right)$ are routed into modes $u, p$ or $w, q$ with probabilities shown in Fig. 1(c). For these terms, the cases

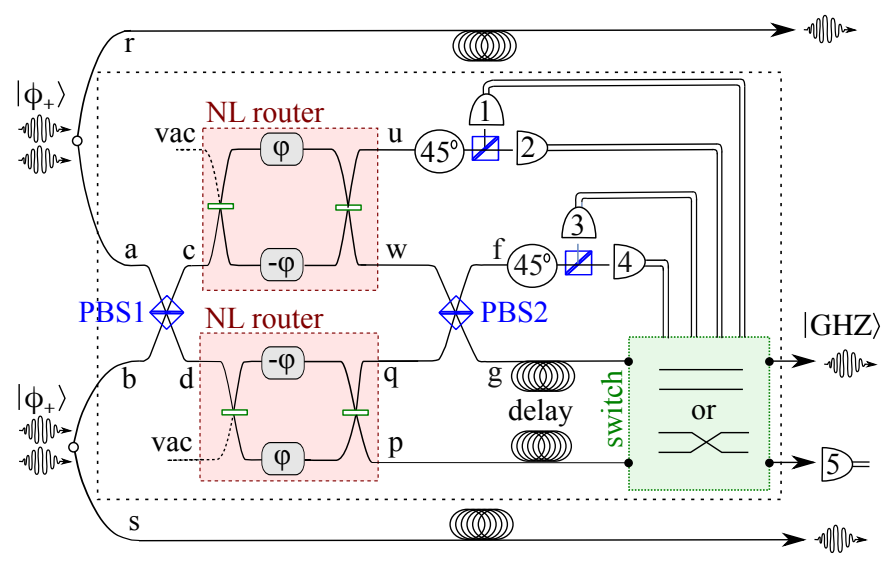

FIG. B.1. To fascillitate the reading of the derivation, we present Fig. 3(a) from the main text also here. 
where both photons are also routed into $w$ or $q$ lead to successful GHZ generation. This is because the latter transform as

$$
\begin{aligned}
& \frac{1}{2}\left(r_{V}^{\dagger} s_{H}^{\dagger} w_{H}^{\dagger} w_{V}^{\dagger}+r_{H}^{\dagger} s_{V}^{\dagger} q_{H}^{\dagger} q_{V}^{\dagger}\right) \stackrel{\text { PBS2 }}{\longrightarrow} \frac{i}{2}\left(r_{V}^{\dagger} s_{H}^{\dagger} g_{H}^{\dagger} f_{V}^{\dagger}+r_{H}^{\dagger} s_{V}^{\dagger} f_{H}^{\dagger} g_{V}^{\dagger}\right) \\
& \stackrel{45^{\circ}}{\longrightarrow} \frac{i}{2 \sqrt{2}}\left[f_{+}^{\dagger}\left(r_{V}^{\dagger} s_{H}^{\dagger} g_{H}^{\dagger}+r_{H}^{\dagger} s_{V}^{\dagger} g_{V}^{\dagger}\right)+f_{-}^{\dagger}\left(r_{V}^{\dagger} s_{H}^{\dagger} g_{H}^{\dagger}-r_{H}^{\dagger} s_{V}^{\dagger} g_{V}^{\dagger}\right)\right] .
\end{aligned}
$$

By scrutinizing the terms in the second line of Eq. B.4, one can see that a measurement of the photon in mode $f$ in the diagonal basis projects the surviving photons onto a GHZ state.

\section{C: Introducing single-photon phase shifts}

\section{Nonlinear router}

In this appendix, we introduce single-photon phase shifts in order to achieve conditional (two-photon) phase shifts with reduced nonlinear loss. In the applications that we study in this paper, we find that this trade-off between single-photon phase shifts and loss leads to a minor improvement in the performance. The results of our analysis are shown in Fig. C.1.

In order to reduce the nonlinear loss, we choose photon frequencies that are detuned from the EIT resonance, such that a photon traveling through the medium acquires a phase shift $\varphi_{1}$ and experiences loss, described by the absorption coefficient $\varepsilon_{1}$. Once a photon generates a Rydberg excitation, a subsequent photon inside the blockade radius effectively experiences an ensemble of two-level atoms and acquires a phase shift $\varphi_{2}$ and loss with absorption coefficient $\varepsilon_{2}$. When neglecting loss, this model produces the following transformation rules for the creation operators in the MZI [Fig. 1(a) in the main text]:

$$
\begin{gathered}
f^{\dagger} \rightarrow e^{i \varphi_{1}} f^{\dagger} \quad, \quad f^{\dagger^{2}} \rightarrow e^{i\left(\varphi_{1}+\varphi_{2}\right)} f^{\dagger^{2}} \\
g^{\dagger} \rightarrow e^{-i \varphi_{1}} g^{\dagger} \quad, \quad g^{\dagger^{2}} \rightarrow e^{-i\left(\varphi_{1}+\varphi_{2}\right)} g^{\dagger^{2}}
\end{gathered}
$$

Our motivation is that by introducing small negative $\varphi_{1}$, one needs a smaller $\varphi_{2}$ to achieve the same conditional phase shift,

$$
\varphi \equiv \varphi_{2}-\varphi_{1}
$$

Since the phase shifts and loss coefficients satisfy the circle relations [Eq. C.12 below], we expect to reduce the total loss

$$
\varepsilon \equiv \varepsilon_{1}+\varepsilon_{2}
$$

Once introducing $\varphi_{1} \neq 0$, one must balance the MZI (by adding a linear phase shift to one of its arm) in order for single photons to exit deterministically through one port. Revisiting Eq. (1) from the main text and introducing single-photon phase shifts, $e^{ \pm \varphi_{1}}$, and an additional phase shift of $e^{i \Delta}$ for mode $f$, we obtain

$$
\begin{aligned}
a^{\dagger} \stackrel{\mathrm{BS} 1}{\longrightarrow} & \frac{1}{\sqrt{2}}\left(e^{i\left(\varphi_{1}+\Delta\right)} f^{\dagger}+i e^{-i \varphi_{1}} g^{\dagger}\right) \stackrel{\Delta=-2 \varphi_{1}}{\longrightarrow} \\
& \frac{e^{-i \varphi_{1}}}{\sqrt{2}}\left(f^{\dagger}+i g^{\dagger}\right) \stackrel{\mathrm{BS} 2}{\longrightarrow} e^{-i \varphi_{1}} u^{\dagger} .
\end{aligned}
$$

With this choice of $\Delta$, the MZI is balanced. Next, let us trace the propagation of a photon pair in the MZI. Revisiting Eq. (3), we find

$$
\begin{gathered}
\left(a^{\dagger}\right)^{2} \stackrel{\mathrm{BS} 1}{\longrightarrow} \frac{1}{2}\left(e^{i \frac{\varphi_{1}+\varphi_{2}+\Delta}{2}} f^{\dagger}+i e^{-i \frac{\varphi_{1}+\varphi_{2}}{2}} g^{\dagger}\right)^{2} \stackrel{\Delta=-2 \varphi_{1}}{\longrightarrow} \\
\frac{e^{-2 i \varphi_{1}}}{2}\left(e^{i \frac{\varphi}{2}} f^{\dagger}+i e^{-i \frac{\varphi}{2}} g^{\dagger}\right)^{2} \stackrel{\mathrm{BS} 2}{\longrightarrow} e^{-2 i \varphi_{1}}\left(w^{\dagger} \sin \frac{\varphi}{2}+u^{\dagger} \cos \frac{\varphi}{2}\right)^{2} .
\end{gathered}
$$

In the second line, we used the definition of $\varphi$ [Eq. C.3] ].

Next, we account for the effect of loss on the probability amplitude of the surviving terms. For brevity, we do not keep track of the loss channels and denote them symbolically by the word "loss" in Eqs. (C7-11) below. The transformation rule [Eq. C.2] ] is modified:

$$
\begin{gathered}
f^{\dagger} \rightarrow \sqrt{1-\tau_{1}} e^{i \varphi_{1}} f^{\dagger}+\text { loss }, \\
g^{\dagger} \rightarrow \sqrt{1-\tau_{1}} e^{-i \varphi_{1}} g^{\dagger}+\text { loss }, \\
f^{\dagger^{2}} \rightarrow \sqrt{\left(1-\tau_{1}\right)\left(1-\tau_{2}\right)} e^{i\left(\varphi_{1}+\varphi_{2}\right)} f^{\dagger^{2}}+\text { loss, } \\
g^{\dagger^{2}} \rightarrow \sqrt{\left(1-\tau_{1}\right)\left(1-\tau_{2}\right)} e^{-i\left(\varphi_{1}+\varphi_{2}\right)} g^{\dagger^{2}}+\text { loss. }
\end{gathered}
$$

Tracing the propagation of photon pairs through the MZI, we find [generalizing Eq. (5) in the main text]

$$
\begin{gathered}
a^{\dagger^{2}} \rightarrow \frac{\sqrt{\left(1-\tau_{1}\right)\left(1-\tau_{2}\right)}}{2} e^{-2 i \varphi_{1}}\left[\cos \varphi\left(w^{\dagger^{2}}-u^{\dagger^{2}}\right)-2 \sin \varphi\left(w^{\dagger} u^{\dagger}\right)\right] \\
-\frac{\left(1-\tau_{1}\right)}{2} e^{-2 i \varphi_{1}}\left(w^{\dagger^{2}}+u^{\dagger^{2}}\right)+\text { loss. }
\end{gathered}
$$

The acquired phase shifts and loss coefficients satisfy the circle relation:

$$
\varphi_{i}^{2}+\left(\frac{\mathrm{OD}}{4}-\frac{\varepsilon_{i}}{2}\right)^{2}=\left(\frac{\mathrm{OD}}{4}\right)^{2},
$$

for $i=1,2$, where $e^{-\varepsilon_{i}}=1-\tau_{i}$.

Equation (C.11) is used in Fig. 1(a) in the main text to compute the probability for a photon pair to exit in modes $w, u$ or both when $\varphi_{1} \neq 0$ (dotted curves). The probabilities can be expressed in terms of $\varphi_{1}, \varphi, \varepsilon_{1}$, and $\varepsilon$. We choose $\varphi_{1}$ and $\varphi$ and express $\varepsilon_{1}\left(\varphi_{1}\right)$ using Eq. C.12 and the overall loss, using

$$
\varepsilon\left(\varphi, \varphi_{1}\right)=\varepsilon_{1}\left(\varphi_{1}\right)+\frac{\mathrm{OD}}{2} \pm \sqrt{\left(\frac{\mathrm{OD}}{2}\right)^{2}-4\left(\varphi+\varphi_{1}\right)^{2}} .
$$

\section{Nonlinear Bell measurement}

Introducing conditional phase shifts for identical photon pairs, the transformation rule for creation operators 
(a)

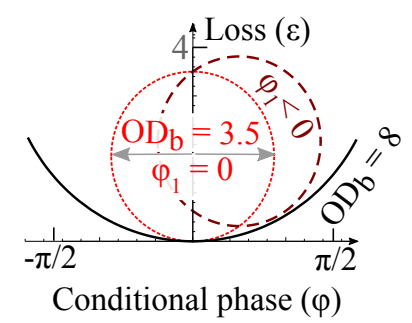

(d) GHZ-state Generation

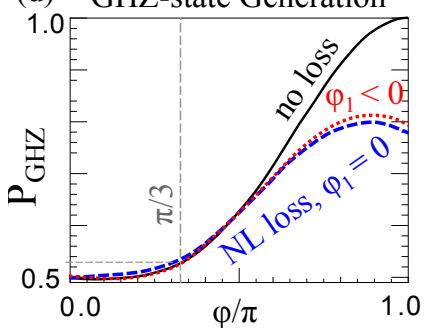

(b)

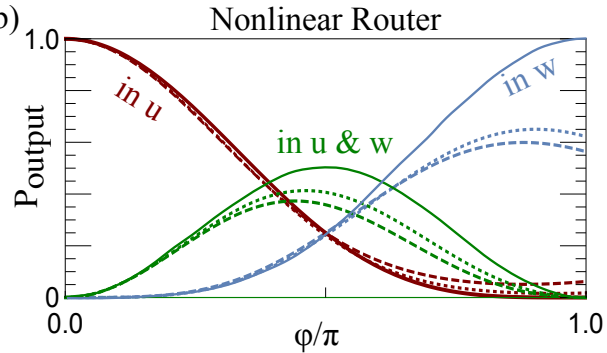

(e)

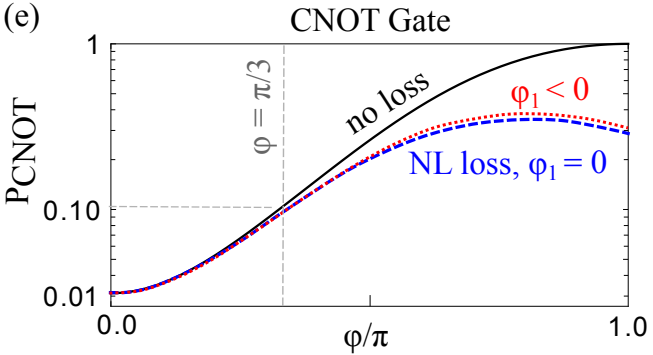

(c)

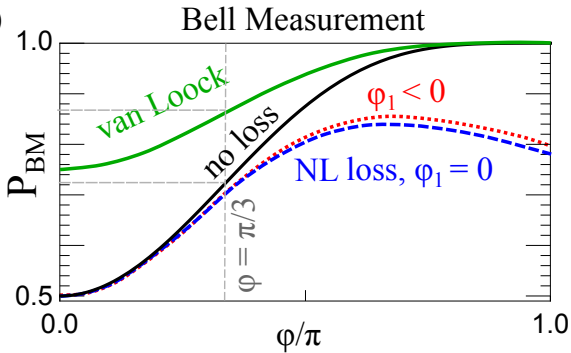

(f)

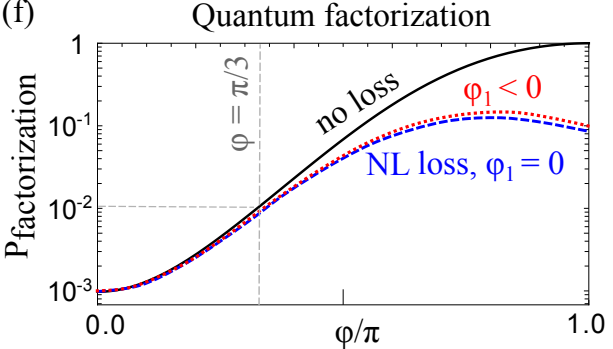

FIG. C.1. Adding a single-photon phase shift, $\varphi_{\mathbf{1}}$, to reduce the nonlinear loss: (a) Conditional phase-loss relation in Rydberg EIT systems, using Eq. C.13). Same as Fig. 1(c), with the addition of the case $\varphi_{1}=-0.5, \mathrm{OD}_{\mathrm{b}}=3.5$ (browndashed curve). (b) Nonlinear router: Probabilities for detection outcomes, $P_{\text {output }}$, for an incoming photon pair. Same as in Fig. 1(b), with the addition of the case $\varphi_{1}=-\frac{\varphi}{11}$, evaluated using Eq. C.11) (dotted curves here and in all of the following subplots in this figure). This value of $\varphi_{1}$ is chosen to optimize the success probability and is used in all remaining subplots. $(\mathrm{c}-\mathrm{f})$ Success probabilities of a nonlinear BM $\left(P_{\mathrm{BM}}\right)$, GHZ-states generation $\left(P_{\mathrm{GHZ}}\right)$, CNOT gate $\left(P_{\mathrm{CNOT}}\right)$, and quantum factorization ( $\left.P_{\text {factorization }}\right)$ as a function of $\varphi$. Same as Figs. 2(c), 3(b), 4(b,c), with the addition of the case $\varphi_{1}<0$.

becomes

$$
\begin{aligned}
f_{ \pm}^{\dagger^{2}} \rightarrow & \sqrt{\left(1-\tau_{1}\right)\left(1-\tau_{2}\right)} e^{i\left(\varphi_{1}+\varphi_{2}\right)} f_{ \pm}^{\dagger^{2}}+\text { loss } \\
& f_{+}^{\dagger} f_{-}^{\dagger} \rightarrow\left(1-\tau_{1}\right) e^{2 i \varphi_{1}} f_{+}^{\dagger} f_{-}^{\dagger}+\text { loss. }
\end{aligned}
$$

Recall that the states $\left|\psi_{-}\right\rangle$and $\left|\phi_{-}\right\rangle$do not contain identical photon pairs in the diagonal basis after the first BS [see Eq. (A.1)]. Therefore, these states can only encounter single-photon loss and the survival probability for each of these states is $\left(1-\tau_{1}\right)^{2}$. Conversely, the states $\left|\psi_{+}\right\rangle$and $\left|\phi_{+}\right\rangle$contain identical photon pairs. By summing the probability of the no-loss terms in Eq. C.11, one finds that the survival probability for each of these states is $\frac{1}{2}\left[\left(1-\tau_{1}\right)^{2}+\left(1-\tau_{1}\right)\left(1-\tau_{2}\right)\right]$. The interpretation of the last result is that either the photons survive two single-photon loss events (in an EIT medium) or they survive a single photon and a second-photon loss (in an effective two-level medium) event. The overall survival probability is

$$
P_{\text {survive }}=\frac{\left(1-\tau_{1}\right)^{2}}{2}+\frac{\left(1-\tau_{1}\right)\left(2-\tau_{1}-\tau_{2}\right)}{4} .
$$

Therefore, the success probability of a BM is

$$
P_{\mathrm{BM}}=P_{\text {survive }}-\frac{1}{8}\left(\sqrt{\left(1-\tau_{1}\right)\left(1-\tau_{2}\right)} \cos \varphi+\left(1-\tau_{1}\right)\right)^{2} .
$$

To account for finite detection efficiency, one needs to multiply this result by $P_{\mathrm{DE}}^{2}$.

\section{Nonlinear GHZ-state generation}

To compute the success probability of our protocol in the presence of $\varphi_{1} \neq 0$, recall that there are two scenarios that produce a GHZ state: (i) When the photon pair leaves PBS1 through different ports and both photons reach the final detectors (avoiding absorption due to linear loss in the MZI), and (ii) When the photons leave PBS1 through the same port and, later, also leave the MZI in modes $w$ or $q$. Adding the probabilities for these scenarios, we obtain

$P_{\mathrm{GHZ}}=\frac{1}{2}\left(1-\tau_{1}\right)^{2}-\frac{1}{8}\left(\sqrt{\left(1-\tau_{1}\right)\left(1-\tau_{2}\right)} \cos \varphi+\left(1-\tau_{1}\right)\right)^{2}$.

\section{D: Selecting optimal phase shifts}

When examining the plots in the main text that show of the success probability versus $\varphi$, a surprising feature emerges: While one would naively expect that $\pi$ phase shifts yield optimal results, it turns out that in all studied applications, the optimal phase is smaller than $\pi$. In Fig. D.1(a), we compute the optimal phase shift, $\varphi_{\text {opt }}$, as a function of the blockaded optical depth, $\mathrm{OD}_{b}$, for or BM and GHZ-state generation protocols. Figure D.1(b) shows the corresponding success probabilities at the optimal phase shifts from (a). The insets show the same data as the main plots on a logarith- 
(a)

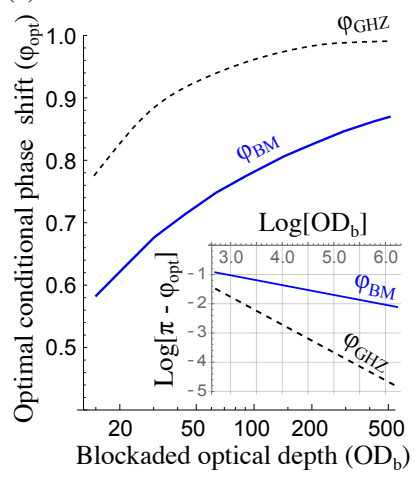

(b)

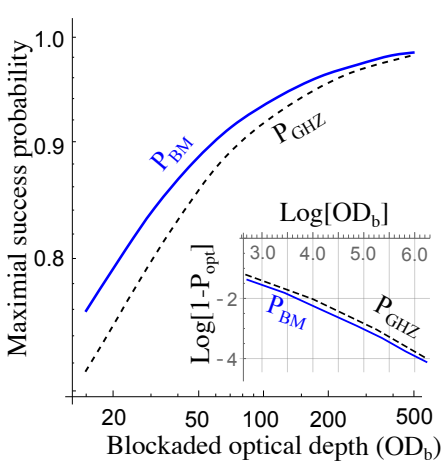

mic scale. One can see that at large optical depths $\left(\mathrm{OD}_{\mathrm{b}}>50\right)$, all curves in the insets are linear. We find that the optimal phases scale as $\varphi_{\mathrm{opt}}-\pi \propto \mathrm{OD}^{\alpha}$, where $\alpha=-1$ for the GHZ-state preparation protocol and $\alpha=-0.3$ for BMs. The infidelity of our protocols scales as $1-P_{\mathrm{opt}} \propto \mathrm{OD}^{\alpha}$, where $\alpha \approx-0.9$ for both protocols.

FIG. D.1. (a) Optimal phase shift as a function of the blockaded optical depth for BMs (blue solid) and for GHZ-state generation (black dashed). (b) The success probabilities at the optimal phases shown in (a). The insets show the same data on a logarithmic scale.
[1] P. Kok, W. J. Munro, K. Nemoto, T. C. Ralph, J. P. Dowling, and G. J. Milburn, "Linear optical quantum computing with photonic qubits," Rev. Mod. Phys. 79, 135 (2007).

[2] J. L. O'brien, A. Furusawa, and J. Vučković, "Photonic quantum technologies," Nat. Photonics 3, 687-695 (2009).

[3] T. Rudolph, "Why I am optimistic about the siliconphotonic route to quantum computing," APL Photonics 2, 030901 (2017).

[4] S. Bartolucci, P. Birchall, H. Bombin, H. Cable, C. Dawson, M. Gimeno-Segovia, E. Johnston, K. Kieling, N. Nickerson, M. Pant, F. Pastawski, T. Rudolph, and C. Sparrow, "Fusion-based quantum computation," arXiv: quant-ph/9705052 (2021).

[5] D. Gottesman and I. L. Chuang, "Demonstrating the viability of universal quantum computation using teleportation and single-qubit operations," Nature 402, 390393 (1999).

[6] R. Raussendorf and H. J. Briegel, "A one-way quantum computer," Phys. Rev. Lett. 86, 5188 (2001).

[7] E. Knill, R. Laflamme, and G. J. Milburn, "A scheme for efficient quantum computation with linear optics," Nature 409, 46-52 (2001).

[8] M. A. Nielsen, "Quantum computation by measurement and quantum memory," Phys. Lett. A 308, 96-100 (2003).

[9] F. Verstraete and J. I. Cirac, "Valence-bond states for quantum computation," Phys. Rev. A 70, 060302(R) (2004).

[10] D. W. Leung, "Quantum computation by measurements," Int. J. Quantum Inf. 2, 33-43 (2004).

[11] H. J. Briegel, D. E. Browne, W. Dür, R. Raussendorf, and M. Van den Nest, "Measurement-based quantum computation," Nat. Phys. 5, 19-26 (2009).

[12] H. J. Briegel and R. Raussendorf, "Persistent entanglement in arrays of interacting particles," Phys. Rev. Lett. 86, 910 (2001).
[13] M. Hein, J. Eisert, and H. J. Briegel, "Multiparty entanglement in graph states," Phys. Rev. A 69, 062311 (2004).

[14] M. A. Nielsen, "Optical quantum computation using cluster states," Phys. Rev. Lett. 93, 040503 (2004).

[15] D. Gottesman, "Stabilizer codes and quantum error correction," arXiv: quant-ph/9705052 (1997).

[16] S. Bravyi and A. Kitaev, "Universal quantum computation with ideal Clifford gates and noisy ancillas," Phys. Rev. A 71, 022316 (2005).

[17] M. Varnava, D. E. Browne, and T. Rudolph, "Loss tolerance in one-way quantum computation via counterfactual error correction," Phys. Rev. Lett. 97, 120501 (2006).

[18] C. M. Dawson, H. L. Haselgrove, and M. A. Nielsen, "Noise thresholds for optical quantum computers," Phys. Rev. Lett. 96, 020501 (2006).

[19] R. Raussendorf, J. Harrington, and K. Goyal, "Topological fault-tolerance in cluster state quantum computation," New J. of Phys. 9, 199 (2007).

[20] A. G. Fowler, M. Mariantoni, J. M. Martinis, and A. N. Cleland, "Surface codes: Towards practical largescale quantum computation," Phys. Rev. A 86, 032324 (2012).

[21] Our work focuses on discrete-variable quantum computation. While large clusters of squeezed states have been demonstrated in continuous-variable quantum computation 92 95, the latter approach faces other challenges that currently hinder its scalability, including the generation of Gottesman-Kitaev-Preskill (GKP) states 96 .

[22] A. Zeilinger, M. A. Horne, H. Weinfurter, and M. Żukowski, "Three-particle entanglements from two entangled pairs," Phys. Rev. Lett. 78, 3031 (1997).

[23] D. E. Browne and T. Rudolph, "Resource-efficient linear optical quantum computation," Phys. Rev. Lett. 95, 010501 (2005).

[24] C.-Y. Lu, X.-Q. Zhou, O. Gühne, W.-B. Gao, J. Zhang, Z.-S. Yuan, A. Goebel, T. Yang, and J.-W. Pan, 
"Experimental entanglement of six photons in graph states," Nat. Phys. 3, 91-95 (2007).

[25] K. Kieling, T. Rudolph, and J. Eisert, "Percolation, renormalization, and quantum computing with nondeterministic gates," Phys. Rev. Lett. 99, 130501 (2007).

[26] M. M. Wilde, F. Spedalieri, J. P. Dowling, and H. Lee, "Alternate scheme for optical cluster-state generation without number-resolving photon detectors," Int. J. Quantum Inf. 5, 617-626 (2007).

[27] P. J. Shadbolt, M. R. Verde, A. Peruzzo, A. Politi, A. Laing, M. Lobino, J. C. F. Matthews, M. G. Thompson, and J. L. O'Brien, "Generating, manipulating and measuring entanglement and mixture with a reconfigurable photonic circuit," Nat. Photonics 6, 45-49 (2012).

[28] X.-L. Wang, L.-K. Chen, W. Li, H.-L. Huang, C. Liu, C. Chen, Y.-H. Luo, Z.-E. Su, D. Wu, Z.-D. Li, H. Lu, Y. Hu, X. Jiang, C.-Z. Peng, L. Li, N.-L. Liu, Y. A. Chen, C. Y. Lu, and J.-W. Pan, "Experimental tenphoton entanglement," Phys. Rev. Lett. 117, 210502 (2016).

[29] D. Istrati, Y. Pilnyak, J. C. Loredo, C. Antón, N. Somaschi, P. Hilaire, H. Ollivier, M. Esmann, L. Cohen, L. Vidro, C. Millet, A. Lemaître, I. Sagnes, A. Harouri, L. Lanco, P. Senellart, and H. S Eisenberg, "Sequential generation of linear cluster states from a single photon emitter," Nature Communications 11, 1-8 (2020).

[30] Y. Li, P. C. Humphreys, G. J. Mendoza, and S. C. Benjamin, "Resource costs for fault-tolerant linear optical quantum computing," Phys. Rev. X 5, 041007 (2015).

[31] C. Schön, K. Hammerer, M. M. Wolf, J. I. Cirac, and E. Solano, "Sequential generation of matrix-product states in cavity QED," Phys. Rev. A 75, 032311 (2007).

[32] N. H. Lindner and T. Rudolph, "Proposal for pulsed on-demand sources of photonic cluster state strings," Phys. Rev. Lett. 103, 113602 (2009).

[33] I. Schwartz, D. Cogan, E. R. Schmidgall, Y. Don, L. Gantz, O. Kenneth, N. H. Lindner, and D. Gershoni, "Deterministic generation of a cluster state of entangled photons," Science 354, 434-437 (2016).

[34] H. Pichler, S. Choi, P. Zoller, and M. D. Lukin, "Universal photonic quantum computation via time-delayed feedback," Proc. Natl. Acad. Sci. 114, 11362-11367 (2017).

[35] L.-M. Duan and H. J. Kimble, "Scalable photonic quantum computation through cavity-assisted interactions," Phys. Rev. Lett. 92, 127902 (2004).

[36] A. Reiserer, N. Kalb, G. Rempe, and S. Ritter, "A quantum gate between a flying optical photon and a single trapped atom," Nature 508, 237-240 (2014).

[37] B. Hacker, S. Welte, G. Rempe, and S. Ritter, "A photon-photon quantum gate based on a single atom in an optical resonator," Nature 536, 193-196 (2016).

[38] S. Rosenblum, A. Borne, and B. Dayan, "Analysis of deterministic swapping of photonic and atomic states through single-photon Raman interaction," Phys. Rev. A 95, 033814 (2017).

[39] O. Bechler, A. Borne, S. Rosenblum, G. Guendelman, O. E. Mor, M. Netser, T. Ohana, Z. Aqua, N. Drucker, R. Finkelstein, Y. Lovsky, R. Bruch, D. Gurovich, E. Shafir, and B. Dayan, "A passive photon-atom qubit swap operation," Nat. Phys. 14, 996-1000 (2018).
[40] J. Borregaard, A. S. Sørensen, and P. Lodahl, "Quantum networks with deterministic spin-photon interfaces," Adv. Quantum Technol. 2, 1800091 (2019).

[41] M. G. A. Paris, M. B. Plenio, S. Bose, D. Jonathan, and G. M. D'ariano, "Optical Bell measurement by Fock filtering," Phys. Lett. A 273, 153-158 (2000).

[42] K. Nemoto and W. J. Munro, "Nearly deterministic linear optical controlled-NOT gate," Phys. Rev. Lett. 93, 250502 (2004).

[43] K. Nemoto and W. J. Munro, "Universal quantum computation on the power of quantum non-demolition measurements," Phys. Lett. A 344, 104-110 (2005).

[44] W. J. Munro, K. Nemoto, and T. P. Spiller, "Weak nonlinearities: a new route to optical quantum computation," N. J. Phys. 7, 137 (2005).

[45] W. J. Munro, K. Nemoto, R. G. Beausoleil, and T. P. Spiller, "High-efficiency quantum-nondemolition singlephoton-number-resolving detector," Phys. Rev. A 71, 033819 (2005).

[46] S. D. Barrett, P. Kok, K. Nemoto, R. G. Beausoleil, W. J. Munro, and T. P. Spiller, "Symmetry analyzer for nondestructive Bell-state detection using weak nonlinearities," Phys. Rev. A 71, 060302(R) (2005).

[47] J. H. Shapiro, "Single-photon Kerr nonlinearities do not help quantum computation," Phys. Rev. A 73, 062305 (2006).

[48] S. G. R. Louis, K. Nemoto, W. J. Munro, and T. P. Spiller, "The efficiencies of generating cluster states with weak nonlinearities," N. J. Phys. 9, 193 (2007).

[49] J. H. Shapiro and M. Razavi, "Continuous-time crossphase modulation and quantum computation," New J. Phys. 9, 16 (2007).

[50] J. Gea-Banacloche, "Impossibility of large phase shifts via the giant Kerr effect with single-photon wave packets," Phys. Rev. A 81, 043823 (2010).

[51] T. M. Stace, S. D. Barrett, and A. C. Doherty, "Thresholds for topological codes in the presence of loss," Phys. Rev. Lett. 102, 200501 (2009).

[52] S. D. Barrett and T. M. Stace, "Fault tolerant quantum computation with very high threshold for loss errors," Phys. Rev. Lett. 105, 200502 (2010).

[53] F. Marsili, V. B. Verma, J. A. Stern, S. Harrington, A. E. Lita, T. Gerrits, I. Vayshenker, B. Baek, M. D. Shaw, R. P. Mirin, and S. N. Nam, "Detecting single infrared photons with $93 \%$ system efficiency," Nat. Photonics 7, 210-214 (2013).

[54] D. V. Reddy, R. R. Nerem, S. W. Nam, R. P. Mirin, and V. B. Verma, "Superconducting nanowire singlephoton detectors with $98 \%$ system detection efficiency at $1550 \mathrm{~nm}$," Optica 7, 1649-1653 (2020).

[55] K.-J. Boller, A. Imamoğlu, and S. E. Harris, "Observation of electromagnetically induced transparency," Phys. Rev. Lett. 66, 2593 (1991).

[56] E. Distante, P. Farrera, A. Padrón-Brito, D. ParedesBarato, G. Heinze, and H. De Riedmatten, "Storing single photons emitted by a quantum memory on a highly excited Rydberg state," Nat. Commun. 8, 1-6 (2017).

[57] D. Tiarks, S. Schmidt-Eberle, T. Stolz, G. Rempe, and S. Dürr, "A photon-photon quantum gate based on Rydberg interactions," Nat. Phys. 15, 124-126 (2019).

[58] O. Lahad and O. Firstenberg, "Induced cavities for photonic quantum gates," Phys. Rev. Lett. 119, 113601 
(2017).

[59] S. Rosenblum, O. Bechler, I. Shomroni, Y. Lovsky, G. Guendelman, and B. Dayan, "Extraction of a single photon from an optical pulse," Nat. Photonics 10, 19-22 (2016).

[60] T. G. Tiecke, J. D. Thompson, N. P. de Leon, L. R. Liu, V. Vuletić, and M. D. Lukin, "Nanophotonic quantum phase switch with a single atom," Nature 508, 241-244 (2014).

[61] T. C. Ralph, I. Söllner, S. Mahmoodian, A. G. White, and P. Lodahl, "Photon sorting, efficient bell measurements, and a deterministic controlled- $Z$ gate using a passive two-level nonlinearity," Phys. Rev. Lett. 114, 173603 (2015).

[62] C. Gerry, P. Knight, and P. L. Knight, Introductory Quantum Optics (Cambridge university press, 2005).

[63] L. Stern, B. Desiatov, I. Goykhman, and U. Levy, "Nanoscale light-matter interactions in atomic cladding waveguides," Nat. Commun. 4, 1-7 (2013).

[64] M. Keil, O. Amit, S. Zhou, D. Groswasser, Y. Japha, and R. Folman, "Fifteen years of cold matter on the atom chip: promise, realizations, and prospects," J. Mod. Opt. 63, 1840-1885 (2016).

[65] J. Kitching, "Chip-scale atomic devices," Appl. Phys. Rev. 5, 031302 (2018).

[66] M. Bajcsy, S. Hofferberth, V. Balic, T. Peyronel, M. Hafezi, A. S. Zibrov, V. Vuletic, and M. D. Lukin, "Efficient all-optical switching using slow light within a hollow fiber," Phys. Rev. Lett. 102, 203902 (2009).

[67] V. Venkataraman, K. Saha, P. Londero, and A. L. Gaeta, "Few-photon all-optical modulation in a photonic band-gap fiber," Phys. Rev. Lett. 107, 193902 (2011).

[68] V. Venkataraman, K. Saha, and A. L. Gaeta, "Phase modulation at the few-photon level for weaknonlinearity-based quantum computing," Nat. Phot. 7, 138 (2013).

[69] Y. Meng, A. Dareau, P. Schneeweiss, and A. Rauschenbeutel, "Near-ground-state cooling of atoms optically trapped $300 \mathrm{~nm}$ away from a hot surface," Phys. Rev. X 8, 031054 (2018).

[70] N. V. Corzo, J. Raskop, A. Chandra, A. S. Sheremet, B. Gouraud, and J. Laurat, "Waveguide-coupled single collective excitation of atomic arrays," Nature 566, 359-362 (2019).

[71] A. Johnson, M. Blaha, A. E. Ulanov, A. Rauschenbeutel, P. Schneeweiss, and J. Volz, "Observation of collective superstrong coupling of cold atoms to a $30-\mathrm{m}$ long optical resonator," Phys. Rev. Lett. 123, 243602 (2019).

[72] N. Matsuda, R. Shimizu, Y. Mitsumori, H. Kosaka, and K. Edamatsu, "Observation of optical-fibre Kerr nonlinearity at the single-photon level," Nat. photonics 3, 95-98 (2009).

[73] A. V. Gorshkov, J. Otterbach, M. Fleischhauer, T. Pohl, and M. D. Lukin, "Photon-photon interactions via Rydberg blockade," Phys. Rev. Lett. 107, 133602 (2011).

[74] There is a factor of 2 difference between our definition of $\varepsilon$ and Ref. [58, since we refer to loss of modal amplitude and Ref. [58] refers to intensity loss.

[75] C. Tresp, C. Zimmer, I. Mirgorodskiy, H. Gorniaczyk, A. Paris-Mandoki, and S. Hofferberth, "Single-photon absorber based on strongly interacting Rydberg atoms," Phys. Rev. Lett. 117, 223001 (2016).

[76] S. Baur, D. Tiarks, G. Rempe, and S. Dürr, "Singlephoton switch based on Rydberg blockade," Phys. Rev. Lett. 112, 073901 (2014).

[77] A. Gaj, A. T. Krupp, J. B. Balewski, R. Löw, S. Hofferberth, and T. Pfau, "From molecular spectra to a density shift in dense Rydberg gases," Nat. Commun. 5, 1-5 (2014).

[78] F. Ewert and P. van Loock, "3/4-efficient Bell measurement with passive linear optics and unentangled ancillae," Phys. Rev. Lett. 113, 140403 (2014).

[79] S. L. Braunstein and A. Mann, "Measurement of the Bell operator and quantum teleportation," Phys. Rev. A 51, R1727 (1995).

[80] This calculation assumes that the nonlinearity is implemented using self-phase modulation, which takes place when two photons of the same polarization travel along the same arm of the MZI. The latter is experimentally achievable in existing Rydberg-EIT platforms [57, 89.

[81] A. Divochiy, F. Marsili, D. Bitauld, A. Gaggero, R. Leoni, F. Mattioli, A. Korneev, V. Seleznev, N. Kaurova, O. Minaeva, G. Gol'tsman, G. L. Konstantinos, M. Benkhaoul, F. Lévy, and A. Fiore, "Superconducting nanowire photon-number-resolving detector at telecommunication wavelengths," Nat. Photonics 2, 302-306 (2008).

[82] E. A. Dauler, A. J. Kerman, B. S. Robinson, J. K. W. Yang, B. Voronov, G. Goltsman, S. A. Hamilton, and K. K. Berggren, "Photon-number-resolution with sub-30-ps timing using multi-element superconducting nanowire single photon detectors," J. Mod. Opt. 56, 364-373 (2009).

[83] D. Sahin, A. Gaggero, Z. Zhou, S. Jahanmirinejad, F. Mattioli, R. Leoni, J. Beetz, M. Lermer, M. Kamp, S. Höfling, and A. Fiore, "Waveguide photon-numberresolving detectors for quantum photonic integrated circuits," Appl. Phys. Lett. 103, 111116 (2013).

[84] M. Endo, T. Sonoyama, M. Matsuyama, F. Okamoto, S. Miki, M. Yabuno, F. China, H. Terai, and A. Furusawa, "Quantum detector tomography of superconducting nanostrip photon-number-resolving detector," arXiv: quant-ph/2102.09712 (2021).

[85] Z.-Y. Liu, Y.-H. Chen, Y.-C. Chen, H.-Y. Lo, P.-J. Tsai, I. A. Yu, Y.-C. Chen, and Y.-F. Chen, "Large crossphase modulations at the few-photon level," Phys. Rev. Lett. 117, 203601 (2016).

[86] W. P. Grice, "Arbitrarily complete Bell-state measurement using only linear optical elements," Phys. Rev. A 84, 042331 (2011).

[87] A. Politi, J. C. F. Matthews, and J. L. O'brien, "Shor's quantum factoring algorithm on a photonic chip," Science 325, 1221-1221 (2009).

[88] Since finite detection efficiency limits our protocols in the same manner as the linear protocols, it does not affect the improvement factor and, hence, not included in the presented equation.

[89] O. Firstenberg, T. Peyronel, Q.-Y. Liang, A. V. Gorshkov, M. D. Lukin, and V. Vuletić, "Attractive photons in a quantum nonlinear medium," Nature 502, 7175 (2013).

[90] R. Finkelstein, G. Winer, D. Z. Koplovich, O. Arenfrid, T. Hoinkes, G. Guendelman, M. Netser, E. Poem, 
A. Rauschenbeutel, B. Dayan, and O. Firstenberg, "Super-extended nanofiber-guided field for coherent interaction with hot atoms," arXiv: 2010.08935 (2020).

[91] X.-L. Pang, A.-L. Yang, J.-P. Dou, H. Li, C.-N. Zhang, E. Poem, D. J. Saunders, H. Tang, J. Nunn, I. A. Walmsley, and X.-M. Jin, "A hybrid quantum memoryenabled network at room temperature," Sci. Adv. 6, eaax1425 (2020).

[92] S. Yokoyama, R. Ukai, S. C. Armstrong, C. Sornphiphatphong, T. Kaji, S. Suzuki, J.-I. Yoshikawa, H. Yonezawa, N. C. Menicucci, and A. Furusawa, "Ultra-large-scale continuous-variable cluster states multiplexed in the time domain," Nat. Photonics 7, 982-986 (2013).

[93] M. Chen, N. C. Menicucci, and O. Pfister, "Experimental realization of multipartite entanglement of 60 modes of a quantum optical frequency comb," Phys. Rev. Lett. 112, 120505 (2014).

[94] C. Reimer, M. Kues, P. Roztocki, B. Wetzel, F. Grazioso, B. E. Little, S. T. Chu, T. Johnston, Y. Bromberg, L. Caspani, D. J. Moss, and R. Morandotti, "Generation of multiphoton entangled quantum states by means of integrated frequency combs," Science 351, 1176-1180 (2016).

[95] W. Asavanant, Y. Shiozawa, S. Yokoyama, B. Charoensombutamon, H. Emura, R. N. Alexander, S. Takeda, J.-I. Yoshikawa, N. C. Menicucci, H. Yonezawa, and A. Furusawa, "Generation of time-domain-multiplexed two-dimensional cluster state," Science 366, 373-376 (2019).

[96] D. Gottesman, A. Kitaev, and J. Preskill, "Encoding a qubit in an oscillator," Phys. Rev. A 64, 012310 (2001). 